\title{
DK-1D: a drift-kinetic simulation tool for modelling the shear Alfvén wave and its interaction with collisionless plasma
}

Article

Accepted Version

Watt, C. E. J. and Rankin, R. (2008) DK-1D: a drift-kinetic simulation tool for modelling the shear Alfvén wave and its interaction with collisionless plasma. Plasma Physics and Controlled Fusion, 50 (7). 074008. ISSN 0741-3335 doi: https://doi.org/10.1088/0741-3335/50/7/074008 Available at https://centaur.reading.ac.uk/32965/

It is advisable to refer to the publisher's version if you intend to cite from the work. See Guidance on citing.

Published version at: http://dx.doi.org/10.1088/0741-3335/50/7/074008

To link to this article DOI: http://dx.doi.org/10.1088/0741-3335/50/7/074008

Publisher: Institute of Physics

All outputs in CentAUR are protected by Intellectual Property Rights law, including copyright law. Copyright and IPR is retained by the creators or other copyright holders. Terms and conditions for use of this material are defined in the End User Agreement.

www.reading.ac.uk/centaur 
Central Archive at the University of Reading

Reading's research outputs online 


\title{
DK-1D: A drift-kinetic simulation tool for modelling the shear Alfvén wave and its interaction with collisionless plasma
}

\author{
C E J Watt and R Rankin \\ Department of Physics, University of Alberta, Edmonton, Alberta, T6G 2G7, Canada \\ E-mail: cwatt@phys.ualberta.ca
}

\begin{abstract}
We present a highly accurate tool for the simulation of shear Alfvén waves (SAW) in collisionless plasma. SAW are important in space plasma environments because for small perpendicular scale lengths they can support an electric field parallel to the ambient magnetic field. Electrons can be accelerated by the parallel electric field and these waves have been implicated as the source of vibrant auroral displays. However, the parallel electric field carried by SAW is small in comparison to the perpendicular electric field of the wave, making it difficult to measure directly in the laboratory, or by satellites in the near-Earth plasma environment. In this paper, we present a simulation code that provides a means to study in detail the SAWparticle interaction in both space and laboratory plasma. Using idealised, smallamplitude propagating waves with a single perpendicular wavenumber, the simulation code accurately reproduces the damping rates and parallel electric field amplitudes predicted by linear theory for varying temperatures and perpendicular scale lengths. We present a rigorous kinetic derivation of the parallel electric field strength for smallamplitude SAW and show that commonly-used inertial and kinetic approximations are valid except for where the ratio of thermal to Alfvén speed is between 0.7 and 1.0. We also present nonlinear simulations of large-amplitude waves and show that in cases of strong damping, the damping rates and parallel electric field strength deviate from linear predictions when wave energies are greater than only a few percent of the plasma kinetic energy, a situation which is often observed in the magnetosphere. The driftkinetic code provides reliable, testable predictions of the parallel electric field strength which can be investigated directly in the laboratory, and will help to bridge the gap between studies of SAW in man-made and naturally occuring plasma.
\end{abstract}

PACS numbers: 94.05.-a 94.30.Aa 52.35.Mw 52.65.Tt 


\section{Introduction}

Shear Alfvén waves (SAW) are an important wave mode in both space and laboratory plasma. They allow the transport of energy and information throughout space plasma, in the solar corona, the solar wind, in planetary magnetospheres and are also important in more distant astrophysical plasma. In the laboratory, early experiments demonstrated that magnetic disturbances can be guided along the magnetic field at the appropriate Alfvén phase velocity, whereas more modern experiments reveal that the detailed wave characteristics of SAW predicted by theory can be reproduced convincingly in the laboratory (see review by Gekelman (1999), and references therein).

There is a growing body of observational evidence which suggests that shear Alfvén waves play a key role in the acceleration of auroral electron acceleration in the Earth's magnetosphere (Keiling et al., 2002; Mende et al., 2003; Semeter et al., 2005), and transport a significant amount of energy from the magnetosphere into the ionosphere and upper atmosphere (Chaston et al., 2007). The analysis of SAW using kinetic theory reveals that field-aligned electron acceleration may be achieved when the perpendicular scale length of the SAW is short in comparison to electron kinetic scale lengths in the plasma, resulting in the generation of a field-aligned component of the electric field (Hasegawa, 1976; Goertz and Boswell, 1979). SAW which travel through plasma with $v_{t h, e} \ll v_{A}$, where $v_{t h, e}$ is the electron thermal speed and $v_{A}$ is the Alfvén speed, will carry a significant parallel electric field when their perpendicular wave number $k_{\perp} \delta_{e} \sim 1$, where $\delta_{e}=c / \omega_{p e}$ is the electron inertial length. Waves fulfilling this criteria are often referred to as inertial SAW, since the parallel electric field is due to electron inertia. In the opposite limit, SAW in plasma with $v_{t h, e} \gg v_{A}$ are sometimes referred to as kinetic SAW, and will carry a significant parallel electric field when $k_{\perp} \delta_{e} v_{t h, e} / v_{A} \sim 1$.

In-situ satellite observations suggest that magnetospheric SAW that are important for auroral acceleration originate in the plasma sheet or plasma sheet boundary layer of the magnetosphere (Wygant et al., 2000, 2002; Dombeck et al., 2005; Keiling et al, 2005), and contribute to electron acceleration at some point along the geomagnetic field between the plasma sheet and the upper atmosphere. The location of this wave-mediated acceleration region is still under debate. Many observations (see review by Mozer et al., (1980) and references therein) suggest that acceleration takes place at an altitude of 1$2 R_{E}$, where the plasma will typically have electron number density $n_{e} \sim 10^{6}-10^{8} \mathrm{~m}^{-3}$, electron temperatures $T_{e} \sim 1 \mathrm{eV}$ and ambient magnetic field strength $B_{0}=1000 \mathrm{nT}$ (Kletzing et al., 1998). This results in plasma with $v_{t h, e} \ll v_{A}$, i.e. in the inertial regime. On the other hand, conditions in the plasma sheet and plasma sheet boundary layer $\left(n_{e} \sim 10^{4}-10^{5} \mathrm{~m}^{-3}, T_{e} \sim 500-3000 \mathrm{eV}\right.$, and $\left.B_{0} \sim 100-400 \mathrm{nT}\right)$ are also conducive to shear Alfven wave electron acceleration (Wygant et al., 2002; Watt and Rankin, 2007b) and researchers have presented observational evidence that the acceleration may begin at higher altitudes $\left(3-4 R_{E}\right)$ (Janhunen et al., 2004, 2006). The warm plasma sheet has $v_{t h, e} \geq v_{A}$, and is typically not warm enough to be in the kinetic regime, but in some intermediate stage where both inertial and kinetic effects are important. 
The parallel electric fields predicted in the inertial and kinetic limits described above have opposite signs, and it has been suggested that for $v_{t h, e} \sim v_{A}$, i.e. in the magnetospheric plasma sheet, the parallel electric field contributions may act to cancel each other out (Chaston et al., 2003a). Subsequent analysis (Shukla and Stenflo, 2004) has suggested that a more rigorous approach is necessary to investigate the behaviour of SAW when $v_{t h} \sim v_{A}$. It is essential that the parallel electric field carried by $\mathrm{SAW}$ in plasma conditions appropriate to the magnetospheric plasma sheet is correctly determined in order to identify the location of auroral electron acceleration.

Detailed observational studies of SAW with short perpendicular scale lengths in naturally occuring space plasma present significant challenges. Spacecraft travel through the plasma at speeds $\sim 10 \mathrm{~km} / \mathrm{s}$, at angles which can be highly oblique to the local magnetic field direction, and often only catch a glimpse of the SAW which race along the geomagnetic field at speeds $v_{A}>1000 \mathrm{~km} / \mathrm{s}$. Accordingly, there has been significant interest in using other methods to study this wave-particle interaction, namely direct laboratory experiments and numerical computer modelling.

Laboratory experiments on shear Alfvén waves require large, dense and highly magnetized plasma. In order to provide results which are easily transferable to space plasma phenomena, the laboratory plasma should be created such that ionization is maximised and particle collisions are minimised. Under these circumstances, laboratory experiments at the Large Plasma Device (LAPD) at the University of California at Los Angeles have determined that the propagation of SAW from a finite, small source region is significantly modified due to the presence of small perpendicular wavelengths introduced by the source (Gekelman et al., 1994; 1997). For plasma with $v_{t h, e} \ll v_{A}$, magnetic disturbances are shown to spread in the perpendicular direction, as predicted by the kinetic theory of inertial SAW (Morales et al., 1994). Gekelman et al . (1994) also presented indirect evidence for a laboratory observation of the parallel electric field associated with SAW in the inertial limit, by showing that the observed wave radiation patterns could only be explained by including kinetic Landau damping into the theoretical model. Recent laboratory experiments have concentrated on possible generation mechanisms for SAW, applicable to space plasma (Van Compernolle et al., $2005 ; 2006)$. Most pertinent to the present work, a study using the LAPD has measured SAW dispersion in the plasma regime $v_{t h, e} \sim v_{A}$ (Kletzing et al., 2003) and shown that the best agreement between theory and experiment is obtained when the fully complex kinetic dispersion relation is used. In this paper, we will use the simulation code to investigate this regime further, using both small-amplitude linear waves and large amplitude waves which show nonlinear effects.

The study of shear Alfvén wave physics is also promoted by numerical modelling. Magnetospheric physicists are largely interested in the possiblity of SAW-mediated electron acceleration, which requires parallel electric fields and therefore short perpendicular scale lengths. Numerical modelling of the shear Alfvén wave-plasma interaction is not straightforward. Kinetic equations must be used to describe the parallel electric wave field, which is strictly forbidden in ideal magnethydrodynamics 
(MHD). On the other hand, the length and time scales of the shear Alfvén waves in collisionless space plasma are many times larger than the Debye length and plasma period which are important in kinetic simulations. Assumptions are therefore required to obtain tractable numerical models. The most straightforward way to study electron acceleration in SAW is to use a modified fluid code to predict the size of the parallel electric field using linear kinetic approximations (Rankin and Tikhonchuk, 1998; Rankin et al., 1999a, 1999b; Streltsov and Lotko, 1999). Specific aspects of the electron accleration mechanism can then be studied by following test-particles as they are influenced by the moving parallel electric field structure (Thompson and Lysak, 1996; Chaston et al., 2000; 2002; 2003a; 2003b; Kletzing, 1994; Kletzing and Hu, 2001; $\mathrm{Su}$ et al., 2004). However, these methods lack self-consistency, and agreement with observations can sometimes only be obtained through application of specific plasma conditions which may prove too restrictive in the Earth's magnetosphere.

Full self-consistent simulations of SAW have been achieved using particle in cell (PIC) simulations (Clark and Seyler, 1999; Génot et al., 2000; 2001; 2004; Tsiklauri et al., 2005; Seyler and Liu, 2007), which demonstrate that nonlinear wave evolution is a key part of the SAW-electron acceleration process. Hybrid codes (drift-kinetic PIC electrons, fluid ions) have also been used to study the SAW-electron interaction (Damiano et al., 2003; 2005; 2007; Swift, 2007). However, PIC simulations suffer from noise and it is difficult to diagnose the strength and form of the parallel electric field which results from the SAW without significant averaging over time and space in the simulation. Direct comparisons between $E_{\|}$from PIC simulations and theory is therefore challenging. Attempts have been made to minimise the numerical noise inherent in particle simulations, but this can yield a simulation which is restricted to certain plasma regimes, e.g. where the wave phase velocity is small compared to the plasma thermal velocity (Lin and Chen, 2001). This hybrid model has been used successfully to study the cross-field propagation of kinetic Alfvén waves in a toroidal magnetic field geometry with plasma conditions appropriate for laboratory experiments (Nishimura et al., 2007). In order to apply such a model to space plasma regimes, it would be necessary to overcome the restriction of small wave phase velocity compared to electron thermal velocity. The simulation developed by Lee et al., (2001) lifts this restriction, employing a sophisticated split-weight scheme to model the gyrokinetic response of both electrons and ions to SAW. This reduced-noise particle simulation can be used over the entire range of plasma beta $\beta=2 \mu_{0} n_{e} k_{B} T_{e} / B_{0}^{2}$, and has been used to model nonlinear electrostatic ion temperature gradient instabilities in conditions appropriate to laboratory plasma experiments (Lin et al., 2007).

For specific studies of the parallel electric field strength and resulting electron acceleration due to electromagnetic SAW, it is desirable to have a self-consistent kinetic code with minimal numerical noise and appropriate assumptions on the length and time scales to allow for a reasonable simulation runtime. We have developed DK-1D, a driftkinetic one-dimensional Eulerian plasma simulation code (Watt et al., 2004) based upon the drift-kinetic treatment [e.g. Catto et al., (1981)]. We neglect the ion response to 
the SAW, and the evolution of the perpendicular wavenumber, in order to maintain a reasonable size of computational domain. However, even with these assumptions, results from DK-1D have successfully been compared against in-situ satellite measurements of electron acceleration, and have provided valuable interpretation of the details of the observations (Watt et al., 2005; 2006). The resonant acceleration of electrons by SAW has been demonstrated to rely upon the strength of the parallel electric field, and the phase velocity of the wave relative to the thermal spread of the electrons in velocity space (Watt and Rankin, 2007a). Here, we demonstrate the accuracy and utility of the plasma simulation for a wide range of plasma parameters, for both small and large amplitude plasma waves. We will show that DK-1D is a valuable tool which can bridge the gap between laboratory investigations and measurements of natural plasma phenomena in space.

We proceed in Section 2 with a description of the governing equations and assumptions used in DK-1D. Section 3 presents results from the simulation code which are used to investigate the damping rates and paralel electric field strength for SAW with different perpendicular scale lengths and wave amplitudes in plasmas with varying $v_{t h, e} / v_{A}$. In Section 4, we present a discussion of these results and the implications for analysing SAW in laboratory experiments and in observations of solar system plasma, before presenting our conclusions in Section 5.

\section{Simulation Code}

For the simulations reported in this paper, we use the drift-kinetic simulation detailed in Watt et al. (2004). The governing equations are derived assuming that the ions carry the perpendicular current of the shear Alfven wave and the electrons carry the parallel current. Other perpendicular electron drifts are neglected. The plasma response in the parallel direction can therefore be fully described using only the equations of electron motion, and ion motion is ignored. The simulation therefore cannot include any finite ion temperature effects. This simulation follows three physical variables on a fixed grid as they evolve in time $t$; the scalar potential $\phi(z)$, where $z$ is the coordinate along the ambient magnetic field direction:

$$
\frac{\partial \phi}{\partial t}=-v_{A}^{2} \frac{\partial A_{\|}}{\partial z}
$$

the electron distribution function $f\left(z, p_{\|}, \mu\right)$, where $p_{\|}=v_{\|}+\left(q_{e} / m_{e}\right) A_{\|}$is the parallel canonical momentum per unit mass and $\mu$ is the magnetic moment,

$\frac{\partial f}{\partial t}+\left(p_{\|}-\frac{q_{e}}{m_{e}} A_{\|}\right) \frac{\partial f}{\partial z}+\left[\frac{q_{e}}{m_{e}}\left\{\left(p_{\|}-\frac{q_{e}}{m_{e}} A_{\|}\right) \frac{\partial A_{\|}}{\partial z}-\frac{\partial \phi}{\partial z}\right\}-\frac{\mu}{m_{e}} \frac{\partial B_{0}}{\partial z}\right] \frac{\partial f}{\partial p_{\|}}=0$,

and finally the parallel component of the vector potential $A_{\|}(z)$ :

$$
A_{\|}=\frac{\mu_{0} q_{e} \int_{-\infty}^{\infty} p_{\|} f d p_{\|}}{k_{\perp}^{2}+\mu_{0} \frac{q_{e}^{2}}{m_{e}} \int_{-\infty}^{\infty} f d p_{\|}} .
$$


We use the parallel canonical momentum $p_{\|}$instead of the parallel velocity $v_{\|}$to avoid including a term of the form $\partial A_{\|} / \partial t$ in the kinetic equation (2). It is very difficult to find a stable explicit algorithm to integrate the distribution function forward in time when it depends upon another temporal derivative so we circumvent the problem by changing the coordinates (Jenko, 2000).

The parallel electric field $E_{\|}(z)$ is calculated as a diagnostic of the simulation, it is not required in any of the three governing equations:

$$
E_{\|}=-\frac{\partial \phi}{\partial z}-\frac{\partial A_{\|}}{\partial t}
$$

In this paper, we simulate the electron response to SAW in a uniform magnetic field, hence the mirror force term [the last term in square parentheses in (2)] is not required, and we can reduce $f$ to a function of $z$ and $p_{\|}$. The electron distribution function is defined on a fixed grid which is uniform in $z$ and $p_{\|}$, respectively. The vector potential $A_{\|}$is defined on the same spatial grid as $f$, whereas the scalar potential $\phi$ occupies grid points half-way between the $A_{\|}$points. A MacCormack algorithm (Horne and Freeman, 2001) is used to evaluate (2), and the inpairs algorithm described by Horne and Freeman (2001) is used to evaluate the moments in (3). $A_{\|}$and $f$ are calculated at integer timesteps $n \Delta t$ and $\phi$ is evaluated at half-timesteps $(n+1 / 2) \Delta t$ using a leapfrog algorithm.

In this paper, we investigate idealized, infinite shear Alfvén waves, hence the boundary conditions in the spatial direction are periodic. The boundary conditions in parallel momentum space for the distribution function assume that the distribution function is Gaussian for values of $\left|p_{\|}\right|$which are greater than the maximum value simulated $p_{\max } \sim 5 v_{t h, e}$. The grid resolution in the spatial direction is $N_{z} \sim 200$ points, whereas the grid resolution in parallel momentum space is $500<N_{p}<7000$ points. Extra fine grid resolution is required when $v_{A} \sim v_{t h, e}$, since the plasma interacts strongly with the wave fields, resulting in perturbations in $f$ which can quickly approach the grid resolution unless it is originally set to be very fine. Too coarse a parallel momentum grid quickly results in numerical instabilities, often referred to as filamentation instabilities in Vlasov-style simulation codes, which quickly swamp the physical solution [see e.g. Klimas (1987)]. However, even with a large number of momentum grid points, each of the simulations reported in this paper takes less than $\sim 24$ hours on a serial computing facility.

\section{Simulation Results}

\subsection{Linear waves}

In this section, we investigate waves with amplitudes such that $\left|q_{e} \phi_{\max }\right| / k_{B} T_{e}$ is very small $(<1 \%)$, where $\phi_{\max }$ is the maximum value of scalar potential in the simulation. By choosing small-amplitude waves, the simulation is guaranteed to follow plasma physics in the linear regime, allowing us to rigorously test and monitor the performance of DK-1D before studying nonlinear and non-idealised wave-particle interactions. 
We avoid any possible mis-application of initial plasma and wave conditions by initialising the wave potentials to zero and driving a propagating wave in the simulation. Plasma behaviour and wave evolution is then studied once the driver has been turned off. A term of the form $-R \omega \sin \left(k_{\|} z-\omega t\right)$ is added to (1), where $k_{\|}=2 \pi / L_{z}, L_{z}$ is the length of the simulation domain, $R$ is a ramping factor (usually $<1$ ), and $\omega$ is the real part of the solution to the warm plasma kinetic dispersion relation:

$$
1+\frac{\omega^{2}-k_{\perp}^{2} v_{A}^{2}}{k_{\perp}^{2} \delta_{e}^{2} k_{\|}^{2} v_{t h}^{2}} Z^{\prime}(\zeta)=0,
$$

where $Z^{\prime}(\zeta)$ is the derivative of the plasma dispersion function (Fried and Conte, 1961) with respect to its argument $\zeta=\omega /\left(k_{\|} v_{t h}\right)$. For all waves studied in this paper, the real frequency is small compared to the ion gyrofrequency.

We will compare damping rates and parallel electric field strength between many simulations with different plasma and wave parameters. The parallel electric field is a diagnostic of the simulation code, and does not directly form part of the governing equations (1-3). We also want to compare the parallel electric field strength determined in the simulations with that predicted by linear kinetic theory. We obtain an expression for the ratio of parallel to perpendicular electric field for a warm electron plasma with cold ions by following the method of Nakamura (2000) (see Appendix for full derivation):

$$
\frac{E_{\|}}{E_{\perp}}=-k_{\perp} k_{\|} \frac{v_{t h}^{2}}{v_{A}^{2}} \delta_{e}^{2} \frac{1}{2[1+\zeta Z(\zeta)]}
$$

Note that the right hand side of this equation will in general have a complex value, since the plasma dispersion function $Z$ is complex.

The evolution of the parallel electric field in a typical small-amplitude wave simulation run is shown in Figure 1. In this case, the ambient magnetic field strength $B_{0}=400 \mathrm{nT}$, the electron number density $n_{e}=3 \times 10^{5} \mathrm{~m}^{-3}$ and the electron temperature $T_{e}=334 \mathrm{eV}$ (hence $v_{t h} / v_{A}=0.68$ ). These values are typical for the plasma sheet of the magnetosphere [see Wygant et al (2000)]. A wave with $k_{\|}=1.2 \times 10^{-7} \mathrm{~m}^{-1}$, $k_{\perp}=5.15 \times 10^{-5} \mathrm{~m}^{-1}$ and $\omega=1.83 \mathrm{rad} / \mathrm{s}$ is driven for three wave periods. Figure 1 (a) shows a contour plot of the parallel electric field as a function of space and time in the simulation. The amplitude of the oscillation grows steadily over three periods until the driver is turned off (this time is indicated by the dashed line in all four panels). Once the driver is turned off, the wave amplitude decreases through Landau damping. Figure 1(b) shows the Fourier transform (in spatial dimension) of the parallel electric field, as a function of time. The enhancement due to the driven wave can be clearly seen at the lower boundary of the figure. Whilst the wave is being driven, we can see there is electric field power at larger values of $k_{\|}$, but this is likely due to the imposed driving function. The enhancements at high $k_{\|}$disappear after the wave driving term has been switched off, and the only significant parallel electric field signal is from the desired wave. Figure 1(c) shows the time evolution of the Fourier amplitude of the driven wave mode $\left(k_{\|}=1.2 \times 10^{-7} \mathrm{~m}^{-1}\right)$. For times after the driver has been turned off, the wave mode decays exponentially. The wave damping rate is calculated by using 
linear regression to fit the slope of the logarithm of the fourier amplitude of the driven mode. Finally, Figure 1(d) shows the ratio of the parallel to perpendicular electric field as a function of time. This ratio is calculated by determining the maximum values of $\left|E_{\|}\right|$and $\left|E_{\perp}\right|$ at each timestep in the simulation. This ratio is highly variable whilst the wave is being driven, before settling down to a quasi-static level later in the simulation. We will compare the ratio $E_{\|} / E_{\perp}$ for different plasma and wave parameters by taking the average value of $E_{\|} / E_{\perp}(t)$ for times after the wave driver has been switched off.

The variation of damping rates and $E_{\|} / E_{\perp}$ as a function of perpendicular scale length is shown in Figure 2, for an ambient magnetic field strength $B_{0}=400 \mathrm{nT}$, electron number density $n_{e}=3 \times 10^{5} \mathrm{~m}^{-3}$ and electron temperature $T_{e}=500 \mathrm{eV}$ (hence $\left.v_{t h} / v_{A}=0.83\right)$. Figure 2(a) shows the damping rates calculated from different simulation results (crosses) and the predicted linear damping rates from solutions to the dispersion relation in (5) (solid line), which are in very good agreement, even for damping rates which are a significant fraction of the real frequancy $\omega=1.9 \mathrm{rad} / \mathrm{s}$. Figure $2(\mathrm{~b})$ shows the ratio $E_{\|} / E_{\perp}$ for the simulation (crosses) compared with the prediction given by (6) (circles), again showing excellent agreement between simulation and theory. The difference between theoretical linear approximations and simulation results for $|\gamma|$ is $<1 \%$, and for $E_{\|} / E_{\perp}$ is $<3 \%$. For all simulation results shown in Figure 2, the ratio of wave potential energy to plasma energy $\left|q_{e} \phi\right| /\left(k_{B} T_{e}\right)<0.002$, ensuring that the simulation is reproducing the linear behaviour of the wave.

We study the effects of varying $v_{t h, e} / v_{A}$ between 0.1 and 10.0 in Figure 3 . This range of values encompasses both the inertial and kinetic regimes. Figure 3(a) shows the simulation damping rates (black '+' signs) compared with the theoretical predictions from the solutions to (5) (red crosses), and just as in Figure 2(a), agreement is excellent. Figure 3(b) shows the simulation $E_{\|} / E_{\perp}$ (black '+' signs) compared with the theoretical predictions from (6). Across the entire range of $v_{t h, e} / v_{A}$, the simulation results agree with the theoretical predictions: the difference between theoretical linear approximations and simulation results for $|\gamma|$ is $<6 \%$, and for $E_{||} / E_{\perp}$ is $<7 \%$. Again, the ratio of wave potential energy to plasma energy is very small for all simulation results used in this figure, $\left|q_{e} \phi\right| /\left(k_{B} T_{e}\right)<0.005$.

\subsection{Nonlinear Waves}

In this section, we study waves with larger amplitudes, to discover whether nonlinear effects change the strength of the parallel electric field associated with shear Alfvén waves. In our first comparison, we study waves with large damping rates, with $v_{t h} / v_{A}=$ $0.83, k_{\perp} \delta_{e}=1.0$ and $|\gamma| / \omega=0.2$. Figure $4(\mathrm{a})$ shows the evolution of the parallel and perpendicular components of the electric field, and the perpendicular component of the magnetic field for the driven wave mode for a simulation with $\left|q \phi_{\max } /\left(k_{B} T_{e}\right)\right|=0.045$ (note that these perpendicular wave field components are calculated from the simulation potentials by multiplying by $k_{\perp}$ ). The wave is driven until $t=7.6 \mathrm{~s}$ (dashed line), after which the perpendicular electric and magnetic field components decay rapidly until 
$t=9.5 \mathrm{~s}$. After this time, the wave amplitudes oscillate in time, as is expected from nonlinear Landau damping (O'Neil, 1965). The parallel electric field evolves differently to the perpendicular perturbations, exhibiting damping until $t=11.7 \mathrm{~s}$. After $t=11.7 \mathrm{~s}$, there is a slight increase in the amplitude of the parallel electric field, but this is a much more subtle effect than seen in the perpendicular field components.

It is important to remember that the parallel electric field is a diagnostic of the simulation code, and is not explicitly required in the governing equations due to the change of variables from parallel velocity to parallel canonical momentum per unit mass (Jenko, 2000).

Figure 4(b) shows the perpendicular electric field evolution from seven simulation runs with different driving rates $R$, labelled with the ratio of wave potential energy to plasma energy at the time when the wave reaches its highest amplitude. The time at which the driver is turned off is indicated by the dashed line. The low amplitude simulations damp at the predicted constant rate. As the wave amplitude is increased, we observe nonlinear effects becoming more and more important, and the wave amplitude does not decay to such low levels. Initial damping rates increase as the wave amplitude is increased. For $\left|q \phi_{\max } /\left(k_{B} T_{e}\right)\right|=0.074$, the wave decays for less than $1.5 \mathrm{~s}$ before being stabilised by nonlinear particle trapping.

Figure 5 shows the related evolution of the ratio of parallel to perpendicular electric field for each of the seven simulations, all on the same vertical scale. We concentrate on the evolution of $E_{\|} / E_{\perp}$ for times $t>7.6 \mathrm{~s}$, i.e. after the driving term has been switched off. The lowest amplitude simulation (shown at the top of the figure) evolves in the same way as the linear simulation run shown in detail in Figure 1. The simulations with large amplitude waves evolve differently from the linear case. The ratio of parallel to perpendicular electric field increases in each case above the linear approximation given by equation (6) $E \| / E_{\perp}=0.005$, in some cases by more than a factor of three. In the lowest five panels in Figure 5, there is a peak in the value of $E_{\|} / E_{\perp}$ which is followed by a gradual drop towards a common asymptotic level, which is less than half that predicted by the linear theory. We can see from Figure 4(a) that the differences in evolution between the parallel and perpendicular electric fields can account for these changes in $E \| / E_{\perp}$ : for the interval between the driver being turned off and the first minimum in $E_{\perp}$, the damping rate of $E_{\|}$is much less than that of $E_{\perp}$, hence the ratio of the two components will increase. As $E_{\perp}$ approaches its first maximum, $E_{\|}$is still decreasing, and so the ratio of the two electric field components will decrease.

We concentrate now on cases with weaker damping, with $v_{t h} / v_{A}=0.83, k_{\perp} \delta_{e}=0.2$ and $|\gamma| / \omega=0.01$. Figure 6 shows the evolution of (a) the amplitude of the parallel electric field of the driven wave mode and (b) $E_{\|} / E_{\perp}$ for three different simulations with different driving rates $R$. The three simulation results shown in this figure have $\left|q \phi_{\max } /\left(k_{B} T_{e}\right)\right|=0.03$ (solid line), $\left|q \phi_{\max } /\left(k_{B} T_{e}\right)\right|=0.08$ (dot-dashed line) and $\left|q \phi_{\max } /\left(k_{B} T_{e}\right)\right|=0.16$ (dashed line), although they are difficult to distinguish, and all follow the same linear evolution seen in Figure 1. Even when the wave potential energy rises to $16 \%$ fo the plasma energy, nonlinear effects do not become important 
in the simulation, and all the wave fields (perpendicular and parallel electric field, and perpendicular magnetic field) decay at the rate predicted by linear theory.

\section{Discussion}

It is important to note that the correct application of (6) is vital when predicting the ratio of $E_{\|} / E_{\perp}$, since the right-hand side of (6) is complex. When making the comparisons in Figures 2(b) and 3(b), (6) was evaluated using measured values of average number density, average temperature, real frequency and damping rate from the simulations, and the input values of $B_{0}, k_{\|}$and $k_{\perp}$ (these are fixed). Figure 7(a) shows the absolute value of $E_{\|} / E_{\perp}$ (solid line), the real part (dashed line) and imaginary part (dot-dashed line) for the plasma parameters investigated in Figure 3. From Figure 7(a), it appears to be necessary to include the full complex kinetic treatment when estimating the parallel electric field strength due to a shear Alfvén wave in warm plasma. This echoes the discovery of Kletzing et al (2003), who demonstrated that measurements of the SAW phase velocity were best described using solutions for the full warm plasma dispersion relation.

However, using the full expression (6) can be unwieldy, and requires detailed knowledge of all the plasma and, more specifically, the wave parameters (complex frequency, parallel and perpendicular wavenumber) in order to evaluate it correctly. This information may be difficult to obtain when studying space observations, and is not always well-defined in numerical models and simulations which do not include full kinetic effects. Analytic approximations to (6) are often used in the inertial (Lysak, 1990):

$$
\left(\frac{E_{\|}}{E_{\perp}}\right)_{i}=-\frac{\delta_{e}^{2}}{1+k_{\perp}^{2} \delta_{e}^{2}} k_{\|} k_{\perp},
$$

and in the kinetic regime:

$$
\left(\frac{E_{\|}}{E_{\perp}}\right)_{k}=\rho_{s}^{2} k_{\|} k_{\perp},
$$

where $\rho_{s}^{2}=\delta_{e}^{2} v_{t h, e}^{2} / v_{A}^{2}$. In one publication (Chaston et al., 2003a), kinetic and inertial effects were combined to produce:

$$
\left(\frac{E_{\|}}{E_{\perp}}\right)_{c}=\frac{k_{\|}}{k_{\perp}}\left(\frac{\left(1+k_{\perp}^{2} \delta_{e}^{2}\right)-\left(1+k_{\perp}^{2} \rho_{s}^{2}\right)}{1+k_{\perp}^{2} \delta_{e}^{2}}\right)
$$

although the two limits are not strictly compatible. Note that in (9), we have taken the limit of cold ions to keep the expression consistent with the simulation conditions used in this paper. Figure $7(\mathrm{~b})$ shows the absolute value of $E_{\|} / E_{\perp}$ obtained from (6) (solid line) compared with the three approximations given above: the inertial approximation (dashed line); kinetic approximation (dot-dashed line) and the 'combined' approximation (grey crosses). From this analysis, it would seem that the full kinetic expression given by (6) is required to calculate the ratio $E_{\|} / E_{\perp}$ only for a small region of parameter space around $v_{t h, e} \sim v_{A}$. This will make the analysis of in 
situ observations more straightforward in some plasma regimes, since wave parameters such as wavenumber and the imaginary part of the frequency are difficult to obtain from single-spacecraft measurements.

When we compare linear to nonlinear behaviour, we see that for strongly damped waves, nonlinear effects are important once the wave potential energy is more than a few percent of the plasma energy. Figure 8 shows the distribution function for two of the cases shown in Figure 4: $\left|q \phi_{\max } /\left(k_{B} T_{e}\right)\right|=0.002$ and $\left|q \phi_{\max } /\left(k_{B} T_{e}\right)\right|=0.045$. Figure $8(\mathrm{a})$ and $8(\mathrm{c})$ show a surface plot of the spatial variation of the distribution function at $t=15 \mathrm{~s}$ for the two different cases; particle trapping due to the propagating waves is clearly seen in Figure $8(\mathrm{c})$ for $2 \times 10^{6}<v_{\|}<4 \times 10^{6} \mathrm{~m} / \mathrm{s}$. Figure $8(\mathrm{~b})$ and $8(\mathrm{~d})$ show the spatially-averaged distribution function $f_{e 0}$ for $v_{\|}>0$ at $t=0$ (thin line) and $t=15 \mathrm{~s}$ (thick line). In Figure $8(\mathrm{~b})$, there is very little evolution of $f_{e 0}$ during the $15 \mathrm{~s}$ interval. In this case, the initial wave energy is small, and the gain of energy by electrons due to Landau damping is not large enough to show up in the plot. On the other hand, the plateau formation associated with Landau damping of the SAW can be seen clearly in the larger amplitude simulation shown in Figure 8(d). The trapping width is estimated using $v_{t} \sim\left(2 q_{e} E_{\|} /\left(m_{e} k_{\|}\right)\right)^{1 / 2}$, and is shown in Figure $8(\mathrm{~d})$ using dashed lines.

The high-frequency shear Alfvén waves observed by Wygant et al. (2002) have an estimated wave potential energy of around $100-4000 \mathrm{~V}$ in the magnetospheric plasma sheet with an electron temperature $T_{e} \sim 2 \mathrm{keV}$. Observations of SAW which are important for electron acceleration therefore indicate that they are large-amplitude nonlinear waves. The detailed DK-1D simulations presented in this study show that linear estimates [e.g. from the analysis by Lysak and Lotko (1996)] are inadequate in this regime, and that full nonlinear simulations are required. It is important to note that the nonlinear behaviour in the DK-1D simulation code results from the response of an electron population with a finite temperature to a large amplitude wave. Other known nonlinear effects are not included in this simulation, such as wave energy transfer to ion plasma waves (Seyler and Liu, 2007). In their study, Seyler and Liu (2007) note that wave breaking and the subsequent excitation of ion cyclotron and ion acoustic waves "requires a sufficient number of resonant electrons" to undergo resonant acceleration and form a field-aligned beam. The particle-in-cell simulations of Seyler and Liu (2007) therefore represent more extreme cases than shown here. The DK-1D results reported in this paper demonstrate that the nonlinear electron response is sufficient to cause a deviation from linear theory predictions even when the wave amplitudes are relatively small and no wave steepening is observed.

\section{Conclusions}

The DK-1D simulation code presented in this paper is a highly accurate nonlinear numerical model which can be used to study shear Alfvén wave behaviour in collisionless plasma. It reproduces the damping rates and parallel electric field strength predicted by 
linear theory over a wide range of plasma conditions, including regimes important for magnetospheric and laboratory plasma physics. For small-amplitude waves in plasma with $v_{t h, e} / v_{A}<0.7$, the parallel electric field calculated using the standard inertial approximation (7) is reasonably accurate, whereas for $v_{t h, e} / v_{A}>1.0$, the parallel electric field calculated using the standard kinetic approximation (8) gives satisfactory values. For linear waves in plasma with $0.7 \leq v_{t h, e} / v_{A} \leq 1.0$, the ratio of parallel to perpendicular electric field must be calculated using the modulus of the complex number returned by the linear equation, which is rigorously derived in the appendix. Any other estimate produces misleading results.

The damping rates and parallel electric field strength of SAW are modified by nonlinear effects when the wave amplitude is increased. Damping is reduced due to particle trapping, and the parallel electric field strength exhibits a more complicated evolution when the wave potential energy is more than a few percent of the plasma energy. Amplitudes of this magnitude are often observed in magnetospheric plasma, demonstrating the need for fully self-consistent nonlinear plasma simulations like DK$1 \mathrm{D}$.

The DK-1D simulation code can be used to study the details of the wave-particle interactions involving SAW, and can provide scaling laws that facilitate analysis of laboratory plasma experiments which elucidate physics governing the natural plasma environment of our solar system. A natural extension of the current DK-1D simulation is to extend the code to two-spatial dimensions using a hybrid method similar to that used by Damiano et al (2003) and Swift (2007). Fluid ion equations can be added to the drift-kinetic equations, which would allow the inclusion of finite temperature ion effects, that are important in laboratory plasma and in some regions of our nearEarth space environment. The advantages of the relatively noise-free DK-1D simulation studies demonstrated in this paper could therefore be extended to the study of more complicated plasma physics processes.

\section{Acknowledgements}

CEJW is funded by the Canadian Space Agency. This work has in part been enabled by the use of Westgrid computing resources, which are funded in part by the Canada Foundation for Innovation, Alberta Innovation and Science, BC Advanced Education, and the participating research institutions.

\section{Appendix: Derivation of $E_{\|} / E_{\perp}$ in warm plasma}

We follow the method of Nakamura (2000), and assume that the system is uniform and linear. The magnetic field is in the $z$-direction and there is no uniform electric field. We will assume cold ions to make the calculation consistent with the simulation results. Perturbations all have the form $\exp [i(\mathbf{k} . \mathbf{r}-\omega t)]$ where $\mathbf{k}$ is the wavevector and $\mathbf{r}=(x, y, z)$. The parallel current density $j_{\|}$and perpendicular current density $j_{\perp}$ may 
be connected through current continuity:

$$
k_{\perp} j_{\perp}+k_{\|} j_{\|}=0
$$

The perpendicular current can be obtained from the polarization current equation:

$$
j_{\perp}=\frac{1}{\mu_{0} v_{A}^{2}} \frac{\partial E_{\perp}}{\partial t}=-\frac{i \omega}{\mu_{0} v_{A}^{2}} E_{\perp},
$$

whereas the parallel current is defined by:

$$
j_{\|}=q_{e} \int_{-\infty}^{\infty} v_{\|} \delta f d v_{\|}
$$

where $\delta f$ is the perturbation in the distribution function due to the waves. The unperturbed part of the distribution function is denoted $f_{0}$. The perturbation in $f$ can be obtained from the linearized Vlasov equation:

$$
\delta f=-i \frac{q_{e}}{m_{e}} \frac{E_{\|}\left(\partial f_{0} / \partial v_{\|}\right)}{\left(\omega-k_{\|} v_{\|}\right)}
$$

Substitute (A.4) into (A.3) to obtain:

$$
j_{\|}=-i \frac{q_{e}^{2}}{m_{e}} \int_{-\infty}^{\infty} d v_{\|} \frac{v_{\|} E_{\|}\left(\partial f_{0} / \partial v_{\|}\right)}{\left(\omega-k_{\|} v_{\|}\right)},
$$

We assume that the unperturbed part of the distribution function can be described by a Maxwellian, hence:

$$
\frac{\partial f_{0}}{\partial v_{\|}}=-2 \frac{n_{e} v_{\|}}{\pi^{1 / 2} v_{t h, e}^{3}} \exp \left[-\frac{v_{\|}^{2}}{v_{t h, e}^{2}}\right] .
$$

Substitute (A.6) into (A.5) and perform a change of variables such that $h=v_{\|} / v_{t h, e}$ :

$$
j_{\|}=-2 i \frac{\omega_{p e}^{2} \epsilon_{0}}{\pi^{1 / 2} v_{t h, e} k_{\|}} E_{\|} \int_{-\infty}^{\infty} d h \frac{h^{2} \exp \left[-h^{2}\right]}{h-\zeta},
$$

where $\zeta=\omega /\left(v_{t h, e} k_{\|}\right)$. Adding $-\zeta^{2}+\zeta^{2}$ to the numerator of the integrand allows us to manipulate the integral into a form which resembles the plasma dispersion function:

$$
j_{\|}=-2 i \frac{\omega_{p e}^{2} \epsilon_{0}}{v_{t h, e} k_{\|}} E_{\|}\left\{\zeta+\zeta^{2} \frac{1}{\pi^{1 / 2}} \int_{-\infty}^{\infty} d h \frac{\exp \left[-h^{2}\right]}{h-\zeta}\right\},
$$

where we have used the standard results:

$$
\int_{-\infty}^{\infty} \exp \left(-\alpha x^{2}\right) d x=\left(\frac{\pi}{\alpha}\right)^{1 / 2}
$$

and:

$$
\int_{-\infty}^{\infty} x \exp \left(-\alpha x^{2}\right) d x=0
$$

Fried and Conte [1961] define the plasma dispersion function as:

$$
Z(\zeta)=\frac{1}{\pi^{1 / 2}} \int_{-\infty}^{\infty} d h \frac{\exp \left[-h^{2}\right]}{h-\zeta}
$$

although note that Nakamura (2000) quotes the definition with an opposite sign. To obtain the ratio of parallel to perpendicular electric fields, we substitute (A.11) into 
(A.8), and then proceed to substitute the expressions we have calculated for $j_{\|}$and $j_{\perp}$ into (A.1). Rearranging this expression, we obtain (6). Note that the expression differs from Nakamura's Equation (5) by a factor of 2 (which arises from the derivative of the Maxwellian distribution function) and by a different sign (which can be explained by the different definitions of the plasma dispersion function).

\section{References}

Catto PJ, Tang WM and Baldwin DE 1981 Plasma Physics 23639

Chaston CC, Carlson CW, Ergun RE and McFadden JP 2000 Phys. Scr. T84 64

Chaston CC, Bonnell JW, Carlson CW, Berthomier M, Peticolas LM, Roth I, McFadden JP, Ergun RE and Strangeway RJ 2002 J. Geophys. Res. 1071413

Chaston CC, Bonnell JM, Carlson CW, McFadden JP, Strangeway RJ and Ergun RE 2003a Geophys. Res. Lett. 301289

Chaston CC, Peticolas LM, Bonnell JW, Carlson CW, Ergun RE, McFadden JP and Strangeway RJ 2003 J. Geophys. Res. 1081091

Chaston CC, Carlson CW, McFadden JP, Ergun RE and Strangeway RJ 2007 Geophys. Res. Lett. 34 L07101

Cheng CZ and Knorr G 1976 J. Comput. Phys. 22330

Clark AE and Seyler CE 1999 J. Geophys. Res. 10417233

Damiano PA, Sydora RD and Samson JC 2003 J. Plasma Phys. 69277

Damiano PA and Wright AN 2005 J. Geophys. Res. 110 A01201

Damiano PA, Wright AN, Sydora RD and Samson JC 2007 Phys. Plasmas 14062904

Dombeck J, Cattell CA, Wygant JR, Keiling A and Scudder J 2005 J. Geophys. Res. 110 A12S90

Fried BD and Conte SD 1961 The Plasma Dispersion Function (Academic, New York)

Gekelman W, Leneman D, Maggs JE and Vincena S 1994 Phys. Plasmas 13775

Gekelman W, Vincena S, Leneman D and Maggs JE 1997 J. Geophys. Res. 1027225

Gekelman W 1999 J. Geophys. Res. 104 14,417

Génot V, Louarn P and Mottez F 2000 J. Geophys. Res. 105 27,611

Génot V, Louarn P and Mottez F 2001 J. Geophys. Res. 106 29,633

Génot V, Louarn P and Mottez F 2004 Ann. Geophys. 222081

Goertz CK and Boswell RW 1979 J. Geophys. Res. 847239

Hasegawa A 1976 J. Geophys Res. 815083

Horne RB and Freeman MP 2001 J. Comp. Phys. 171182

Janhunen P, Olsson A, Hanasz J, Russell CT, Laakso H and Samson JC 2004 Ann. Geophys. 222213

Janhunen P, Olsson A, Russell CT and Laakso H 2006 Sp. Sci Rev. 12289

Jenko F 2000 Comput. Phys. Commun. 125196

Keiling A, Wygant JR, Cattell C, Peria W, Parks G, Temerin M, Mozer FS, Russell CT and Kletzing CA 2002 J. Geophys. Res. 1071132

Keiling A, Parks GK, Wygant JR, Dombeck J, Mozer FS, Russell CT, Streltsov AV and Lotko W 2005 J. Geophys. Res. 110 A10S11

Kletzing CA 1994 J. Geophys. Res. 99 11,095

Kletzing CA and Hu S 2001 Geophys. Res. Lett. 28693

Kletzing CA, Mozer FS and Torbert RB 1998 J. Geophys. Res. 103 14,837

Kletzing CA, Bounds SR, Martin-Hiner J, Gekelman W and Mitchell C 2003 Phys. Rev. Lett. 90035004

Klimas AJ 1987 J. Comput. Phys. 68202

Lee WW, Lewandowski JLV, Hahm TS and Lin Z 2001 Phys. Plasmas 84435

Lin Z and Chen L 2001 Phys. Plasmas 81447

Lin Z, Nishimura Y, Xiao Y, Holod I, Zhang WL and Chen L 2007 Plasma Phys. Contr. Fusion 49 B163 
Lysak RL and Lotko W 1996 J. Geophys. Res. 1015085

Mende SB, Carlson CW, Frey HU, Peticolas LM and Østgaard N 2003 J. Geophys. Res. 1081344

Morales GJ, Loritsch RS and Maggs JE 1994 Phys. Plasmas 13765

Mozer FS, Cattell CA, Hudson MK, Lysak RL, Temerin M and Torbert RB 1980 Sp. Sci. Rev. 27155

Nakamura T 2000 J. Geophys. Res. 105 10,729

Nishimura Y, Lin Z and Wang WX 2007 Phys. Plasmas 14042503

O’Neil T 1965 Phys. Fluids 82255

Rankin R and Tikhonchuk VT 1998 J. Geophys. Res. 103, 20,419

Rankin R, Samson JC, Tikhonchuk VT and Voronkov I 1999a J. Geophys. Res. 1044399

Rankin R, Samson JC and Tikhonchuk VT 1999b Geophys. Res. Lett. 263601

Semeter J, Heinselman CJ, Sivjee GG, Frey HU and Bonnell JW 2005 J. Geophy. Res. 110 A11310

Seyler CE and Liu K 2007 J. Geophys. Res. 112 A09302

Shukla PK and Stenflo L 2004 Geophys. Res. Lett. 31 L03810

Su YJ, Jones ST, Ergun RE and Parker SE 2004 J. Geophys. Res. 109 A11201

Swift DW 2007 J. Geophys. Res. 112 A01204

Thompson BJ and Lysak RL 1996 J. Geophys. Res. 1015359

Tsiklauri D, Sakai JI and Saito S 2005 Astronomy and Astrophysics 4351105

Van Compernolle B, Gekelman W, Pribyl P and Carter TA 2005 Geophys. Res. Lett. 32 L08101

Van Compernolle B, Gekelman W and Pribyl P 2006 Phys. Plasmas 13092112

Watt CEJ and Rankin R 2007a J. Geophys. Res. 112 A04214

Watt CEJ and Rankin R 2007b in press, Adv. Sp. Res. doi: 10.1029/j/asr/2007.03.030

Watt CEJ, Rankin R and Marchand R 2004 Phys. Plasmas 111277

Watt CEJ, Rankin R, Rae IJ and Wright DM 2005 J. Geophys. Res. 110 A10S07

Watt CEJ, Rankin R, Rae IJ and Wright DM 2006 Geophys. Res. Lett. 33 L02106

Wygant JR et al. 2000 J. Geophys. Res. 10518,675

Wygant JR et al. 2002 J. Geophys. Res. 1071201 


\section{Figure captions}

Figure 1. (a) Evolution of $E_{\|}$as a function of distance along the simulation domain; (b) Evolution of the spatial discrete fourier transform of $E_{\|}$in the simulation; (c) Evolution of the driven wave mode amplitude; (d) Evolution of the ratio $E_{\|} / E_{\perp}$. Driving term in simulation is turned off after three periods, indicated by the dashed line. Simulation has $B_{0}=400 \mathrm{nT}, n_{e}=3 \times 10^{5} \mathrm{~m}^{-3}$ and $T_{e}=334 \mathrm{eV}$ (hence $\left.v_{t h} / v_{A}=0.68\right)$.

Figure 2. (a) Damping rates from theory (solid line) and simulation (black crosses) as a function of perpendicular wavenumber; (b) $E_{\|} / E_{\perp}$ from (6) (circles) and simulation (crosses) as a function of perpendicular wavenumber.

Figure 3. (a) Damping rates from theory (red crosses) and simulation (black ' + ' signs) as a function of the ratio of thermal speed to Alfvén speed; (b) $E_{\|} / E_{\perp}$ from (6) (red crosses) and simulation (crosses) as a function of the ratio of thermal speed to Alfvén speed.

Figure 4. (a) Evolution of the perpendicular magnetic field, and perpendicular and parallel electric field components derived from simulation potentials for a simulation with $\left|q_{e} \phi\right| / k_{B} T_{e}=0.045$. (b) Evolution of driven wave mode from seven simulations with different driving rate $R$ for a case of strong damping $(|\gamma| / \omega=0.2)$. Each line represents a different simulation and is labelled with the maximum value of $\left|q_{e} \phi\right| /\left(k_{B} T_{e}\right)$ attained during the simulation run. In both (a) and (b), the dashed line indicates the time at which the driving term is switched off.

Figure 5. Evolution of $E_{\|} / E_{\perp}$ from simulations with different driving rate $R$ for a case of strong damping $(|\gamma| / \omega=0.2)$. The maximum value of $q \phi /\left(k_{B} T_{e}\right)$ obtained during each simulation run is indicated to the right of the figure. Each panel shows the same vertical scale.

Figure 6. Evolution of (a) driven wave mode and (b) $E_{\|} / E_{\perp}$ from simulations with different driving rate $R$ for a case of weak damping $(|\gamma| / \omega=0.01)$. The maximum value of $q \phi /\left(k_{B} T_{e}\right)$ attained during the three simulations shown here was 0.03 (solid line), 0.08 (dot-dashed line) and 0.16 (dashed line) although the evolution in each case is very similar. 
Figure 7. (a) Absolute (solid line), real (dashed line) and imaginary (dot-dashed line) parts of $E_{\|} / E_{\perp}$ as given by (6). (b) $E_{\|} / E_{\perp}$ given by (6) - solid line; (7) - dashed line; (8) - dot-dashed line; and (9) - grey crosses.

Figure 8. (a) Surface plot of the distribution function at $t=15 \mathrm{~s}$ during a simulation with $q \phi /\left(k_{B} T_{e}\right)=0.002$; (b) $f_{e 0}$ at $t=0$ (thin line) and $t=15 \mathrm{~s}$ for the same simulation; (c) Surface plot of the distribution function at $t=15 \mathrm{~s}$ during a simulation with $q \phi /\left(k_{B} T_{e}\right)=0.045$; (b) $f_{e 0}$ at $t=0$ (thin line) and $t=15 \mathrm{~s}$ for the same simulation. 

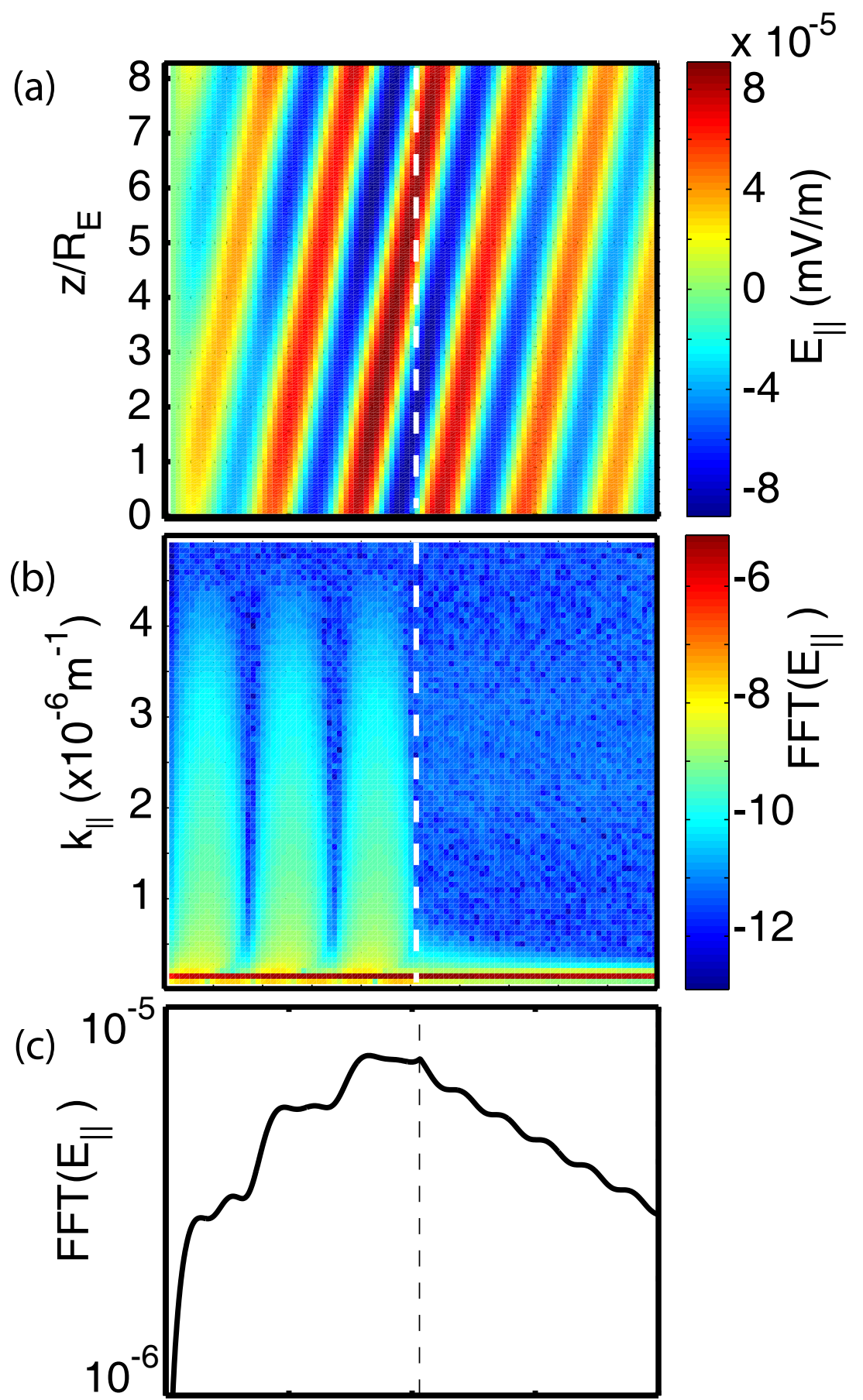

(d)

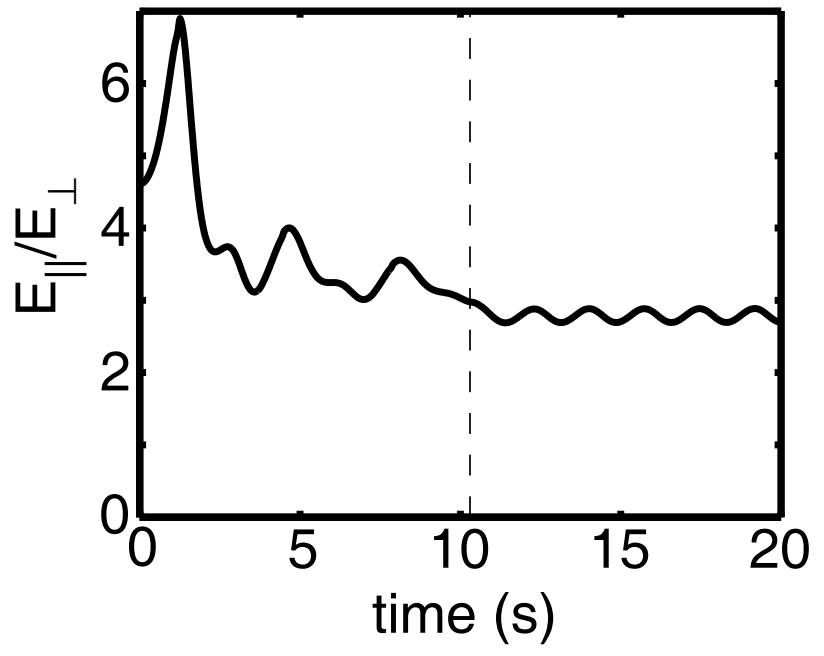




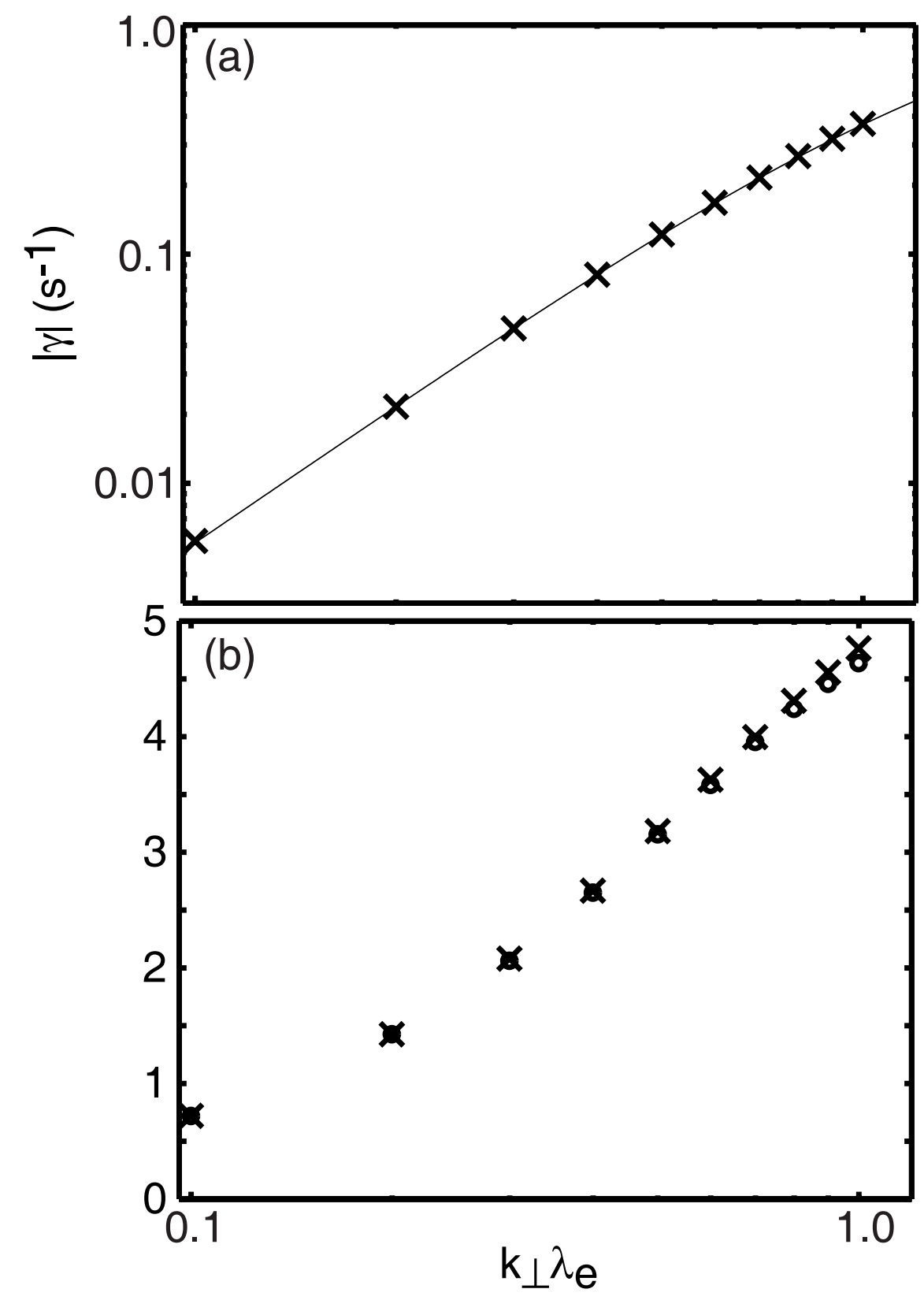




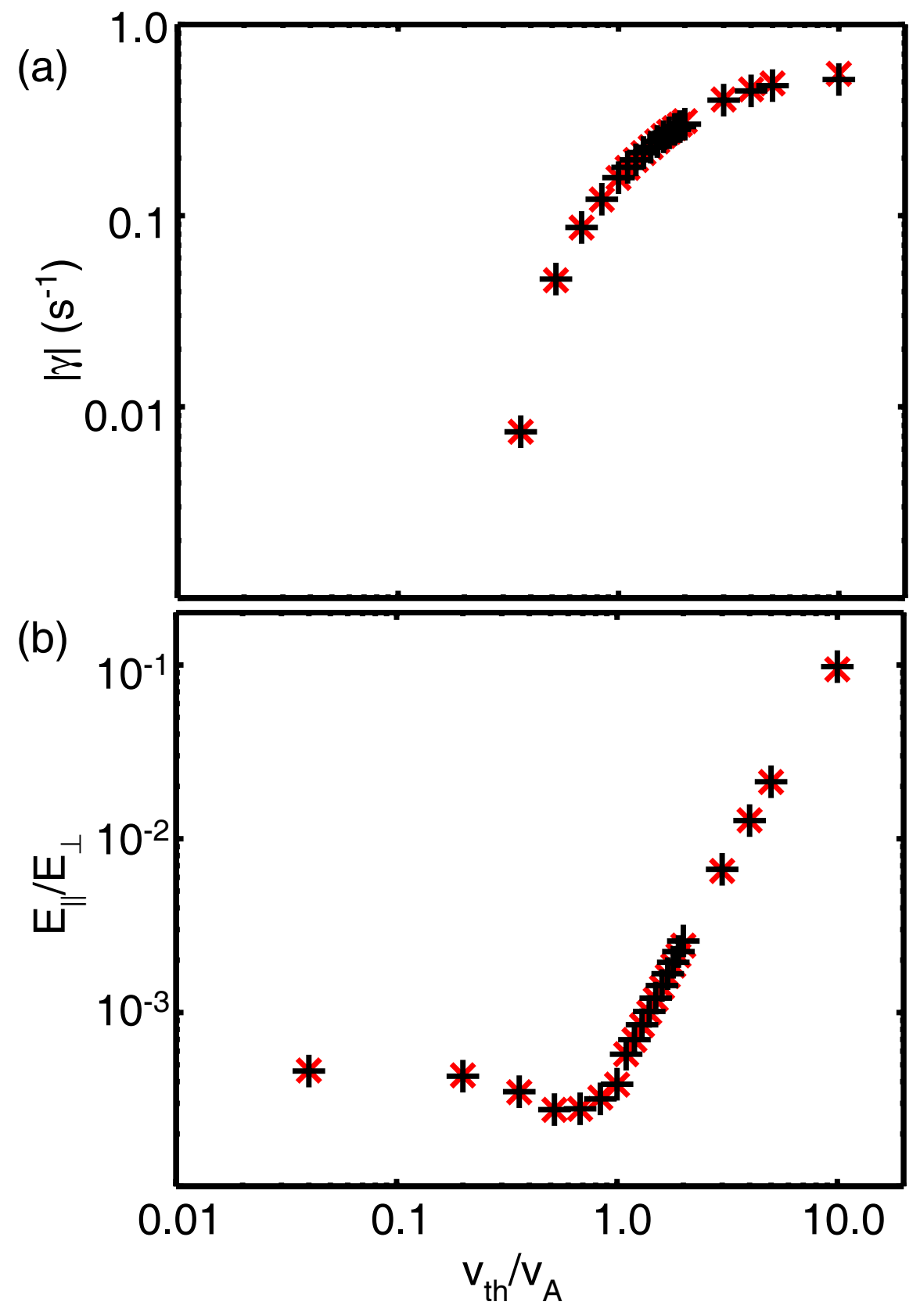



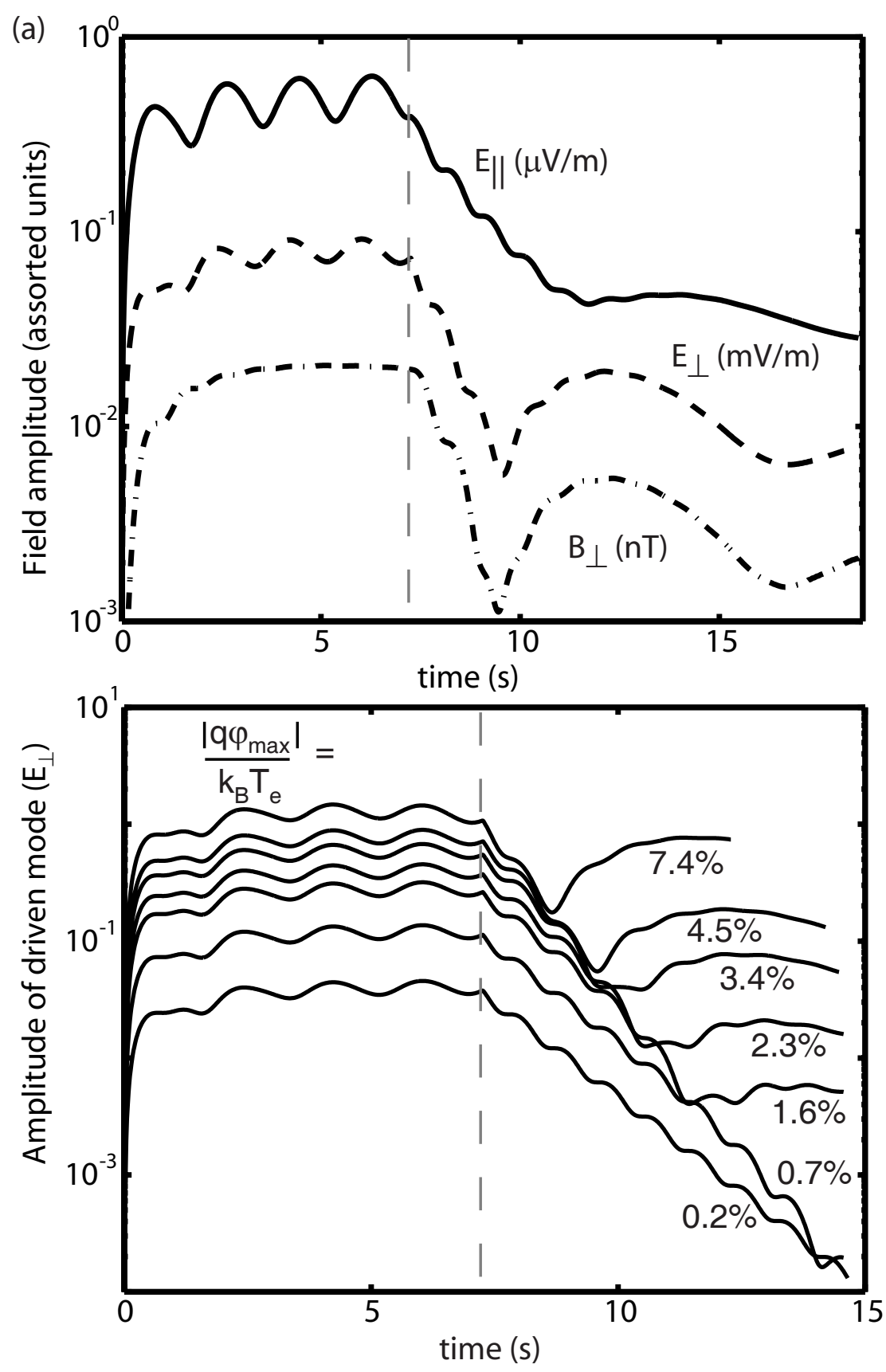


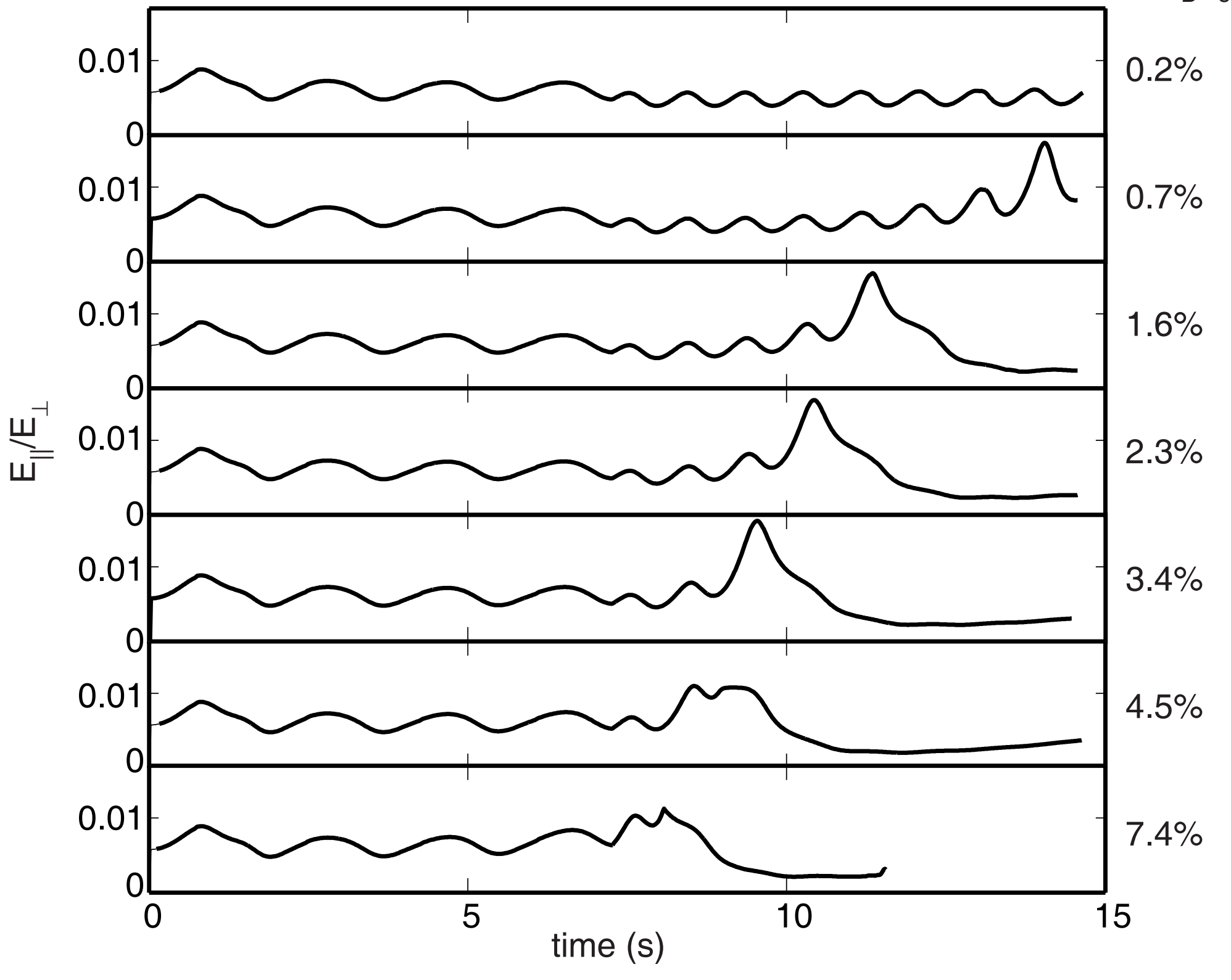



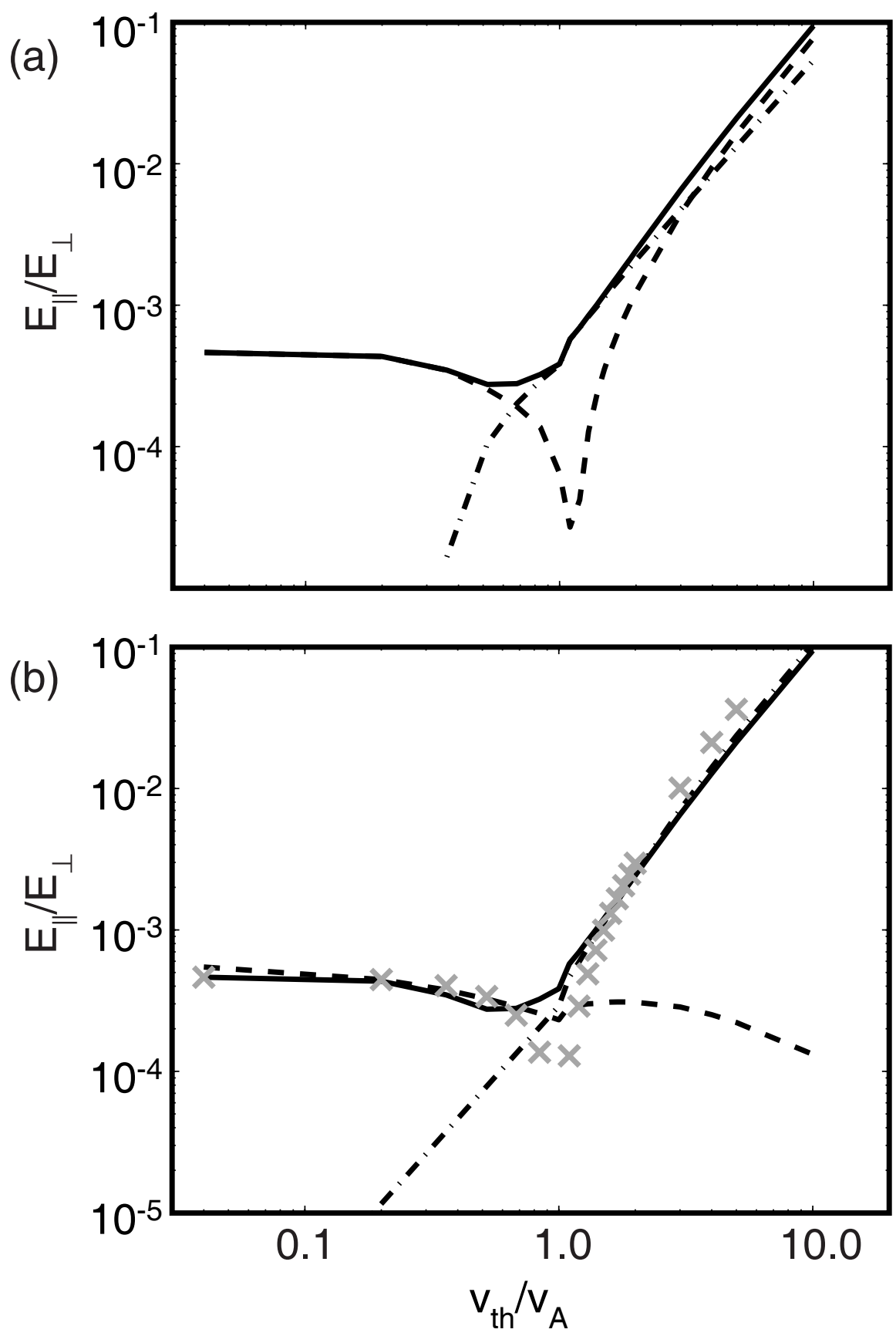


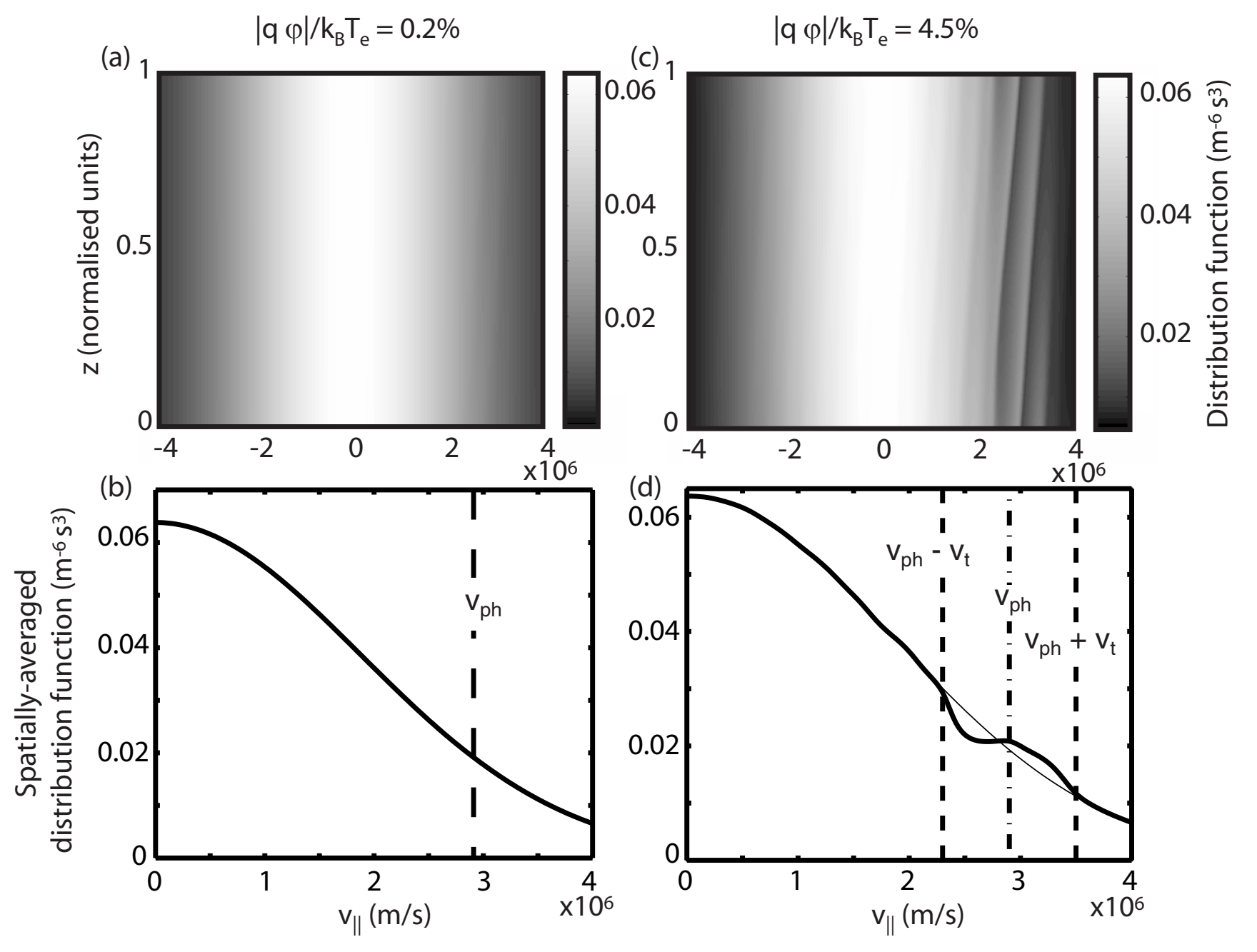

